\title{
DESCONFORTO OSTEOMUSCULAR E QUALIDADE DE VIDA DE MULHERES EM DIFERENTES FASES DA GESTAÇÃO
}

Valeria Andrea Alegria Larrua, Jessica Jessica Ribeiro, Letícia Endsfeldz Teixeira, Nathália Savian Elisa Bizetti Pelai, Alessandra Madia Montovani, Andressa Carvalho Viscone, Mariana Romanholi Palma, Edna Maria do Carmo, Cristina Elena Prado Teles Fregonesi

Curso de Fisioterapia da Universidade Estadual Paulista Júlio de Mesquita Filho - Faculdade de Ciências e Tecnologia FCT/UNESP. E-mail: v.larrua@hotmail.com

\section{RESUMO}

Durante a gestação, o corpo passa por intensas mudanças. O aumento constante de peso causa uma sensação de sobrecarga, fadiga e desconfortos. Mudanças físicas durante o 3o trimestre afetarão as atividades e habilidades da gestante, interferindo na qualidade de vida. O objetivo do estudo foi avaliar a percepção de desconforto osteomuscular, a qualidade de vida e a dor em diferentes fases da gestação comparadas a mulheres não grávidas. Participaram do estudo 49 mulheres entre 18 e 35 anos, divididas em: G1, do 3ㅇa ao 6o mês de gestação; G2, do 7으 ao 9으 mês de gestação; e G3, grupo controle. Foram aplicados o Questionário Nórdico de Sintomas Osteomusculares, o Questionário de Qualidade de Vida SF-36 e a Escala Visual Análoga. Constatou-se que a qualidade de vida é diminuída no 30 trimestre de gestação (G2). No G3 a prevalência de sintomas foi na região cervical, ombros e joelhos.

Palavras-chave: Desconforto; gravidez; qualidade de vida; dor lombar; postura.

\section{INTRODUÇÃO}

No período da gestação, o corpo passa por uma intensa mudança e a mulher se depara com uma nova imagem corporal. Começam então, as mudanças físicas, em que o corpo se modifica para o acolhimento deste bebê nos próximos nove meses ${ }^{(1)}$. Durante esse período também há um grande envolvimento emocional e psicológico da mãe. Os medos aumentam, surgem dúvidas quanto à capacidade de cuidar de seu bebê corretamente, se será uma boa mãe, se irá amá-lo. É um período de adequação em que a rotina da futura mãe será completamente alterada, pois deverá dedicar $100 \%$ de sua atenção ao bebê, reorganizar seu tempo e sua energia $^{(2)}$.

O crescimento da barriga ocasionará um aumento na lordose lombar, podendo causar dor na região e interferir na qualidade de vida, pois pode levar à incapacidades motoras, má qualidade do sono, depressão, fadiga e diminuição no desempenho de atividades domésticas, trabalho e lazer $^{(3,4)}$.

Entre as alterações no corpo da mulher durante a gestação, encontramos a mudança de posição do útero; aumento de peso e das mamas causando sensação de dor; dispneia, pois o 
aumento da cavidade abdominal comprime o diafragma; aumento da frequência respiratória e cardíaca; alterações de pressão arterial; hiperventilação; náuseas e vômitos; constipação intestinal; aumento do volume sanguíneo; e o aumento da pressão sobre a bexiga, que leva à uma maior frequência miccional ${ }^{(5,6,7)}$.

A gestante também apresenta alterações musculoesqueléticas que irão causar-lhe desconforto, pois afetarão sua marcha e postura ${ }^{(8)}$. A alteração da biomecânica leva a uma instabilidade e maior probabilidade de quedas por desequilíbrio ${ }^{(9)}$.

As mudanças no corpo durante o terceiro trimestre afetarão as atividades e habilidades do dia-a-dia da gestante, comprometendo a qualidade de vida desta, pois dependendo do grau de desconfortos, ela perde parte de sua independência física ${ }^{(1)}$.

\section{OBJETIVO}

Este estudo visa identificar e verificar o desconforto musculoesquelético, a qualidade de vida e a dor em diferentes fases da gestação para que futuramente tais dados possam contribuir para elaboração de tratamentos preventivos e específicos, oferecendo para essa população uma melhora na qualidade de vida.

\section{METODOLOGIA}

Trata-se de um estudo observacional transversal, desenvolvido no Laboratório de Estudos Clínicos em Fisioterapia (LECFisio) da Faculdade de Ciências e Tecnologia (FCT) - Universidade Estadual Paulista (UNESP), Campus de Presidente Prudente.

Participaram do estudo 49 voluntárias do gênero feminino com faixa etária de 18 a 35 anos. Estas passaram por uma avaliação inicial para coleta de dados pessoais e antropométricos: nome, idade, tempo de gestação, altura, peso e índice de massa corporal (IMC). Foi também aferida a pressão arterial sistólica (PAS) e diastólica (PAD), frequência cardíaca (FC) e saturação de oxigênio (Sat O2). Segundo o Ministério da Saúde, realizar atividade física regularmente ajuda no controle da pressão arterial, reduzindo o risco de doenças cardiovasculares, facilita o controle de peso e o pós-natal, e indica quando há necessidade de interromper a atividade em caso de sensação de cansaço ${ }^{(10)}$.

As voluntárias foram divididas em três grupos: G1, com 15 gestantes do terceiro ao sexto mês de gestação; G2, com 17 gestantes do sétimo ao nono mês de gestação; e G3, com 17 mulheres não grávidas. Todas as participantes do estudo assinaram o "Termo de Consentimento 
Livre e Esclarecido", cujos procedimentos adotados obedecem aos princípios éticos para pesquisa clínica da Declaração de Helsinque e as diretrizes e normas regulamentadoras de pesquisas, envolvendo seres humanos, contidas na Resolução 466/2012 do Conselho Nacional de Saúde. Esse estudo esta inserido no projeto "A influência da cinesioterapia nos parâmetros clínicos e funcionais de gestantes", o qual está de acordo com as orientações do Comitê de Ética em Pesquisa da FCT/UNESP (processo n69/2011).

Os desconfortos osteomusculares foram verificados por meio do Questionário Nórdico de Sintomas Osteomusculares em que voluntárias relataram a ocorrência de sintomas em diversas regiões anatômicas considerando os 12 meses e/ou os sete dias precedentes à entrevista, bem como a ocorrência de afastamento das atividades rotineiras no último ano ${ }^{(11)}$. Para verificar a qualidade de vida foi utilizada a Versão Brasileira do Questionário de Qualidade de Vida SF-36 (Medical OutcomesStudy 36- Item Short-Form Health Survey) ${ }^{(12)}$. Já para quantificar a dor utilizouse a Escala Visual Análoga (EVA), numerada de 0 a 10, identificando a intensidade da dor sentida no momento ${ }^{(13)}$.

\section{RESULTADOS}

Com relação aos dados pessoais e antropométricos houve diferença significativa somente para a semana gestacional entre G1 e G2 $(p<0,05)$, o que mostra a homogeneidade entre os grupos conforme a Tabela 1. A Tabela 2 mostra os valores de PAS, PAD, FC e Sat02 dos grupos em média e desvio padrão. Não houve diferença significativa para estes dados entre os grupos.

Tabela 1. Valores de Idade, altura, peso, índice de massa corpórea (IMC) e semana gestacional expressos em média e desvio padrão.

\begin{tabular}{lcccc}
\hline \multicolumn{1}{c}{ Variáveis } & $\begin{array}{c}\text { Grupo 1 } \\
(\mathbf{n = 1 5})\end{array}$ & $\begin{array}{c}\text { Grupo 2 } \\
(\mathbf{n = 1 7})\end{array}$ & $\begin{array}{c}\text { Grupo 3 } \\
(\mathbf{n = 1 7})\end{array}$ & Valores de $\boldsymbol{p}$ \\
\hline Idade (anos) & $23,93 \pm 9,98$ & $27,44 \pm 5,89$ & $25,53 \pm 3,08$ & 0,4000 \\
Altura (m) & $1,59 \pm 0,06$ & $1,61 \pm 0,07$ & $1,61 \pm 0,06$ & 0,5609 \\
Peso (kg) & $64,77 \pm 12,76$ & $72,06 \pm 14,80$ & $61,76 \pm 11,89$ & 0,5890 \\
IMC & $25,78 \pm 5,68$ & $27,52 \pm 4,66$ & $23,78 \pm 4,53$ & 0,1203 \\
Semana & $21,13 \pm 3,78$ & $33,06 \pm 3,99$ & NA & $0,0400^{*}$ \\
Gestacional & & & &
\end{tabular}

*valor de $p<0,05$. 
Tabela 2. Valores de pressão arterial sistólica (PAS) e diastólica (PAD), frequência cardíaca (FC) e saturação de oxigênio (Sat02) dos Grupos 1, 2 e Controle expressos em média e desvio padrão.

\begin{tabular}{lcccc}
\hline \multicolumn{1}{c}{ Variáveis } & $\begin{array}{c}\text { Grupo 1 } \\
(\mathbf{n = 1 5})\end{array}$ & $\begin{array}{c}\text { Grupo 2 } \\
(\mathbf{n = 1 7 )}\end{array}$ & $\begin{array}{c}\text { Grupo 3 } \\
(\mathbf{n = 1 7 )}\end{array}$ & Valores de $\boldsymbol{p}$ \\
\hline PAS (mmHg) & $102 \pm 9,41$ & $99,41 \pm 17,84$ & $103,5 \pm 12,72$ & 0,1204 \\
PAD (mmHg) & $62,31 \pm 9,27$ & $65 \pm 12,65$ & $66 \pm 7,37$ & 0,5604 \\
FC (bpm) & $81,53 \pm 12,93$ & $84,70 \pm 12,05$ & $84,25 \pm 7,94$ & 0,1305 \\
SatO2 (\%) & $98,66 \pm 0,49$ & $98,41 \pm 0,79$ & $98,70 \pm 0,59$ & 0,5705 \\
\hline
\end{tabular}

Observou-se que a qualidade de vida na gestante é mais afetada no 30 trimestre de gestação (G2). Porém houve diferença estatística somente para os domínios Capacidade Funcional (CF), Aspectos Físicos (AF), Dor e Aspectos Emocionais (AE) conforme a Tabela 3.

Tabela 3. Valores correspondentes aos domínios Capacidade Funcional (CF), Aspectos Físicos (AF), Dor, Estado Geral de Saúde (EGS), Vitalidade (Vit), Aspectos Sociais (AS), Aspectos Emocionais (AE) e Saúde Mental (SM) do questionário SF-36 expressos em média e desvio padrão.

\begin{tabular}{lccc}
\hline Domínios & Grupo $\mathbf{1}(\mathbf{n = 1 5})$ & Grupo $\mathbf{2}(\mathbf{n = 1 7})$ & Grupo 3 ( $\mathbf{n}=\mathbf{1 7})$ \\
\hline CF & $72,67 \pm 13,99 a^{*}$ & $56,47 \pm 20,97 a^{*}$ & $95 \pm 7,91 b^{*}$ \\
AF & $51,67 \pm 37,16 a^{*}$ & $41,18 \pm 42,33 a^{*}$ & $88,23 \pm 26,69 b^{*}$ \\
Dor & $53,53 \pm 16,27 a^{*}$ & $48,12 \pm 21,82 a^{*}$ & $76,23 \pm 17,49 b^{*}$ \\
EGS & $78,67 \pm 17,33 a$ & $75,18 \pm 24,16 a$ & $81,53 \pm 12,25 a$ \\
Vit & $54,67 \pm 15,40 a$ & $50,59 \pm 18,10 a$ & $60,53 \pm 13,64 a$ \\
AS & $72,5 \pm 29,21 a$ & $72,79 \pm 25,09 a$ & $83,09 \pm 17,64 a$ \\
AE & $56,19 \pm 31,40 a^{*}$ & $49,01 \pm 44,28 a *$ & $84,3 \pm 26,68 b^{*}$ \\
SM & $69,87 \pm 21,37 a$ & $66,59 \pm 23,95 a$ & $72,71 \pm 11,69 a$ \\
\hline
\end{tabular}

*Letras diferentes na mesma linha implicam em diferença estatística, sendo $p<0,05$.

Os desconfortos osteomusculares foram verificados através do Questionário Nórdico de Sintomas Osteomusculares. Na Tabela 4 estão representados os valores de frequência e porcentagem dos sintomas, dor, dormência, formigamento ou desconforto, considerando os últimos 12 meses; considerando os últimos 7 dias; considerando a necessidade de redução de atividades normais no trabalho, serviço doméstico ou passatempos, por conta dos desconfortos, durante os últimos 12 meses. 
Tabela 4: Valores expressos em forma de frequência (n) e porcentagem (\%) dos desconfortos musculoesqueléticos entre os grupos, adquiridos por meio do questionário Nórdico.

\begin{tabular}{|c|c|c|c|c|c|c|c|}
\hline & & \multicolumn{2}{|c|}{$\begin{array}{l}\text { Grupo } 1 \\
(n=15)\end{array}$} & \multicolumn{2}{|c|}{$\begin{array}{l}\text { Grupo } 2 \\
(n=17)\end{array}$} & \multicolumn{2}{|c|}{$\begin{array}{l}\text { Grupo } 3 \\
(n=17)\end{array}$} \\
\hline & & $\mathbf{n}$ & $\%$ & $\mathbf{n}$ & $\%$ & $\mathbf{n}$ & $\%$ \\
\hline \multirow[t]{3}{*}{ Pescoço e cervical } & $12 \mathrm{~m}$ & 3 & 20 & 3 & 17,65 & 15 & 82,25 \\
\hline & 7 dias & 5 & 33,33 & 3 & 17,65 & 12 & 70,58 \\
\hline & Redução & 0 & 0 & 1 & 5,88 & 0 & 0 \\
\hline \multirow[t]{3}{*}{ Ombros } & $12 \mathrm{~m}$ & 1 & 6,66 & 5 & 29,41 & 7 & 46,66 \\
\hline & 7 dias & 2 & 13,33 & 1 & 5,88 & 5 & 29,41 \\
\hline & Redução & 1 & 6,66 & 0 & 0 & 0 & 0 \\
\hline \multirow[t]{3}{*}{ Cotovelos } & $12 \mathrm{~m}$ & 1 & 6,66 & 0 & 0 & 0 & 0 \\
\hline & 7 dias & 3 & 20 & 1 & 5,88 & 0 & 0 \\
\hline & Redução & 0 & 0 & 0 & 0 & 0 & 0 \\
\hline \multirow[t]{3}{*}{ Antebraços } & $12 \mathrm{~m}$ & 1 & 6,66 & 2 & 11,75 & 1 & 5,88 \\
\hline & 7 dias & 3 & 20 & 1 & 5,88 & 0 & 0 \\
\hline & Redução & 0 & 0 & 0 & 0 & 0 & 0 \\
\hline \multirow[t]{3}{*}{ Punho, mãos e dedos } & $12 \mathrm{~m}$ & 13 & 86,66 & 5 & 29,41 & 5 & 29,41 \\
\hline & 7 dias & 5 & 33,33 & 3 & 17,65 & 3 & 17,65 \\
\hline & Redução & 2 & 13,33 & 0 & 0 & 0 & 0 \\
\hline \multirow[t]{3}{*}{ Dorsal } & $12 \mathrm{~m}$ & 10 & 66,66 & 10 & 58,88 & 7 & 46,66 \\
\hline & 7 dias & 11 & 73,33 & 10 & 58,88 & 5 & 29,41 \\
\hline & Redução & 3 & 20 & 0 & 0 & 0 & 0 \\
\hline \multirow[t]{3}{*}{ Lombar } & $12 \mathrm{~m}$ & 13 & 86,66 & 9 & 52,94 & 11 & 64,70 \\
\hline & 7 dias & 14 & 93,33 & 13 & 76,47 & 10 & 58,88 \\
\hline & Redução & 4 & 26,66 & 3 & 17,65 & 0 & 0 \\
\hline \multirow[t]{3}{*}{ Quadril e coxas } & $12 \mathrm{~m}$ & 3 & 20 & 7 & 46,66 & 1 & 5,88 \\
\hline & 7 dias & 8 & 53,33 & 3 & 17,65 & 1 & 5,88 \\
\hline & Redução & 0 & 0 & 3 & 17,65 & 0 & 0 \\
\hline \multirow[t]{3}{*}{ Joelhos } & $12 \mathrm{~m}$ & 1 & 6,66 & 2 & 11,75 & 3 & 17,65 \\
\hline & 7 dias & 5 & 33,33 & 1 & 5,88 & 1 & 5,88 \\
\hline & Redução & 1 & 6,66 & 0 & 0 & 0 & 0 \\
\hline \multirow[t]{3}{*}{ Tornozelos e pé } & $12 \mathrm{~m}$ & 1 & 6,66 & 2 & 11,75 & 3 & 17,65 \\
\hline & 7 dias & 3 & 20 & 1 & 5,88 & 2 & 11,75 \\
\hline & Redução & 1 & 6,66 & 1 & 5,88 & 1 & 5,88 \\
\hline
\end{tabular}

\section{DISCUSSÃO}

De acordo com os resultados do questionário nórdico, pode-se inferir que para G1 a predominância de sintomas foi nos cotovelos, mãos, coluna dorsal e lombar, joelhos e pés. O G2 teve uma prevalência de sintomas, porém com menor frequência, na cervical, antebraço, quadril e coxas.

Os resultados para G1 e G2 se devem as adaptações do corpo para acolher o bebê. A partir da 12 a semana ocorre a mudança de posição do útero, que se torna um órgão abdominal e pode 
aumentar até 150 vezes o seu tamanho e 20 vezes o seu peso ${ }^{(5,7)}$. O aumento de peso irá intensificar os sintomas de fadiga e sensação de sobrecarga na região baixa do abdômen e a sacroilíaca $^{(7)}$. A protrusão da barriga e a frouxidão dos ligamentos contribuem para o aumento da lordose lombar, levando a um desconforto que pode ser irradiado para as pernas ${ }^{(3)}$.

No grupo controle (G3), a prevalência de dor foi observada na região cervical, ombros e joelho. Este grupo foi composto por mulheres não grávidas, estudantes ou funcionárias de uma determinada empresa. Segundo estudos, atualmente existem fatores negativos que interferem na qualidade de vida e desempenho devido às más posturas duradouras, adquiridas e inadequadas no posto de trabalho, sobrecarga emocional e física, e também por consequência de movimentos repetitivos $^{(14)}$.

Como observado, a qualidade de vida na gestante é diminuída entre o 7o e 9o mês de gestação, isto é, no ultimo trimestre gestacional. Estudos mostram que a dor lombar interfere significativamente nas atividades de vida diária, habilidades físicas e de trabalho, pois se manifesta durante a noite e pode causar insônia. A lombalgia é muito comum na população em geral, mas em mulheres grávidas o aparecimento desse sintoma é 14 vezes maior e surge principalmente entre o 4ㅇ e 70 mês de gestação ${ }^{(15)}$. As gestantes deste estudo apresentaram uma porcentagem de $93,33 \%$ (G1) e 76,47\% (G2) para dor lombar nos últimos 7 dias.

\section{CONCLUSÃO}

Conclui-se que a qualidade de vida é afetada e apresenta-se diminuída na gestante, comparado a mulheres não grávidas, principalmente no terceiro trimestre de gestação.

Este trabalho identificou a presença de desconfortos musculoesqueléticos e redução da qualidade de vida na gestante. Portanto, mostra-se importante a elaboração e intervenções de tratamento fisioterapêutico específico para esse tipo de população.

\section{REFERÊNCIAS}

1. VIDO, M. B. Qualidade de vida na gravidez. 2006. Dissertação (Mestrado em Enfermagem) Universidade de Guarulhos, Guarulhos, 2006.

2. DORNELLES, L. M. N.; LOPES, R. C. S. Será que eu consigo levar essa gestação até o fim? A experiência materna da gestação no contexto da reprodução assistida. Estudos de Psicologia, v. 28, n. 4, p. 489-499, out/dez, 2011. http://dx.doi.org/10.1590/S0103166X2011000400009 
3. NOVAES, F. S.; SHIMO, A. K. K.; LOPES, M. H. B. M. Lombalgia na gestação. Revista Latino Americana de Enfermagem, v. 14, n. 4, p. 620-624, jul/ago, 2006.

4. MARTINS, R. F.; E SILVA, J. L. P. Prevalência de dores nas costas na gestação. Revista da Associação Médica Brasileira, v. 51, n. 3, p. 144-147, 2005. http://dx.doi.org/10.1590/S0104-42302005000300014

5. GAZANEO, M. M.; DE OLIVEIRA, L. F. Alterações posturais durante a gestação. Escola de Educação Física e Desportos, UFRJ. Laboratório de Biomecânica. Revista Brasileira de Atividade Física \& Saúde, v. 3, n. 2, 1998.

6. FEITOSA, D. C. A.; DA SILVA, M. G.; PARADA, C. M. G. de. L. Acurácia do exame de urina simples para diagnóstico de infeç̧ões do trato urinário em gestantes de baixo risco. Revista Latino Americana de Enfermagem, v. 17, n. 4, jul/ago, 2009.

7. SPIER, M. As alterações musculoesqueléticas e as queixas de dor e desconforto em gestantes adolescentes. 2009. Monografia (Bacharel em Fisioterapia) - Centro Universitário Feevale, Novo Hamburgo, 2009.

8. BARBOSA, C. M. de. S.; DA SILVA, J. M. N.; DE MOURA, A. B. Correlação entre o ganho de peso e a intensidade da dor lombar em gestantes. Revista Dor, v. 12, n. 3, p. 205-208, 2011. http://dx.doi.org/10.1590/S1806-00132011000300002

9. RIBAS, S. I.; GUIRRO, E. C. O. Análise da pressão plantar e do equilíbrio postural em diferentes fases da gestação. Revista Brasileira de Fisioterapia, v. 11, n. 5, p. 391-396, set/out, 2007.

10. Manual Técnico Pré-Natal e Puerpério. Série Direitos Sexuais e Direitos Reprodutivos, BrasíliaDF. Caderno no 5, p 96, 2006.

11. PINHEIRO, F. M.; TRÓCCOLI, B. T.; DE CARVALHO, C. V. Validação do questionário Nórdico de sintomas osteomusculares como medida de morbidade. Revista Saúde Pública, v. 36, n. 3, p. 307312, 2002. http://dx.doi.org/10.1590/S0034-89102002000300008

12. FERNANDES, I. I. B.; DE VASCONCELOS, K. C.; DA SILVA, L. L. L. Análise da qualidade de vida segundo o Questionário SF-36 nos funcionários da Gerência de Assistência Nutricional (GAN) da Fundação Santa Casa de Misericórdia do Para. 2009. Monografia (Bacharel em Fisioterapia) Centro de Ciências Biológicas e da Saúde da Universidade da Amazônia. Belém, 2009.

13. CARAVIELLO, E. Z.; WASSERSTEIN, S.; CHAMLIAN, T. R.; MASIERO, D. Avaliação da dor e função de pacientes com lombalgia tratados com um programa de Escola de Coluna. ACTA Fisiátrica, v.12, n. 1, p. 11-14. 2005.

14. ABRAHÃO, P. N; DUARTE, M. S. Incidência e prevalência de dores e funcionários administrativos de uma instituição particular de ensino superior do município do Rio de Janeiro, 2007.

15. RAMOS, A. V. B., DE ALMEIDA, C. S. A gestação no segundo trimestre de usuárias da Clínica de Saúde da Mulher e o papel da Fisioterapia. Revista inspirar movimento \& saúde, v. 4, n. 21, nov/dez, 2012. 